\title{
Improving Anti-Fouling Properties of Polyamide Forward Osmosis Membrane by Grafting Poly Ethylenimine and Silver Nanoparticles
}

\author{
Bo Xu ${ }^{a}$, Gang Chen ${ }^{b,}{ }^{*}$, Jin Wang ${ }^{c}$, Manhong Huang ${ }^{d}$ \\ College of Environmental Science and Engineering, Donghua University, Shanghai, China \\ a1053009817@qq.com, *,b cheng@dhu.edu.cn, cwangjin901224@163.com, \\ ${ }^{d}$ huangmanhong@dhu.edu.cn
}

\begin{abstract}
Keywords: Forward osmosis, Poly ethylenimine modification, Anti-fouling, Cationic surfactant (DTAC), Silver nanoparticles (Ag).
\end{abstract}

\begin{abstract}
In this work, the effect of grafting of poly ethylenimine (PEI) on the antifouling performance of an aromatic polyamide forward osmosis membrane was investigated. Results showed that PEI modified membrane showed a good anti-contaminant property in treating wastewater containing cationic surfactant (DTAC). Furthermore, the silver nanoparticles were also introduced on the membrane surface to improve membrane anti-fouling resistance. The silver nanoparticles modified membranes showed excellent antibacterial properties.
\end{abstract}

\section{Introduction}

Forward osmosis technology was widely used in seawater desalination [1-3], surfactant wastewater treatment [4] and other fields because of its low energy consumption, simple operation and high water recovery ratio. Polyamide composite forward osmosis membranes have a wide range of applications in the treatment of high salt-containing wastewater due to their high salt rejection and wide $\mathrm{pH}$ range.

However, when treating wastewater, the membrane surface is often contaminated by positively charged substances such as cationic surfactants, thereby lowering the performance of the membrane. Studies have confirmed that the reason of membrane fouling in the forward osmosis treatment process is related to the negative charge of the membrane surface [5-7]. Therefore, it is necessary to modify the surface of the polyamide forward osmosis membrane without impairing the performance of the membrane and improve the membrane's resistance to contamination.

This study intends to use PEI as the main membrane surface charged modified material to prepare two kinds of PEI modified membranes to improve the membrane surface anti-adhesion ability and antibacterial ability [8]. The contact angle, forward osmosis performance, reverse osmosis performance, and anti-fouling and anti-bacterial characteristics were investigated.

\section{Experiment}

\subsection{Material.}

Polysulfone (PSf, P-3500) was used to prepare support layer of the membrane. N,N-dimethylacetamide (DMAc, analytical grade) and n-hexane (analytical grade) were used as solvents, polyethylene glycol (PEG400, analytically pure) was used as a porogen, both purchased from Sinopharm Chemical Reagent Co., Ltd.; trimesoyl chloride (TMC, >98\%) and m-phenylenediamine (MPD, 99\%) purchased from Sigma was used as interfacial polymerization monomer; polyethylenimine (PEI, $\mathrm{Mw}=70000,50 \%$ aqueous solution) was a charged modification material, purchased from Aladdin Reagent (China) Co., Ltd.; Dodecyl trimethyl ammonium chloride (DTAC) was used as a cationic surfactant purchased from Adamas-beta Reagents Co., Ltd. Silver nanoparticles are antibacterial materials and were purchased from Nanjing Xianfeng Nanomaterials Technology Co., Ltd. 


\subsection{Membrane Fabrication.}

PSf, DMAc, and PEG400 were at a mass ratio of 18:74:8. MPD and TMC were used as the reaction monomers to conduct interfacial polymerization to form a composite membrane. PEI aqueous solution was added to the control membrane to prepare a PEI modified membrane. The PEI-AgNPs modified membrane was obtained by PEI modified membrane soaking in the silver nanocolloids solutions for $15 \mathrm{~h}$ [9].

\subsection{Membrane Characterization.}

The forward osmosis membranes modification were characterized as follows: surface charge analysis (zeta potential), hydrophilicity analysis (static contact angle), and micro-morphology analysis (SEM). All membranes were placed in a dry sample bag and stored at room temperature for testing.

The pure water permeability coefficient, $A$, the salt permeability coefficient $B$, and the salt rejection, $R$, of the composite forward osmosis membrane were measured in the cross-flow reverse osmosis mode. The experiments were conducted using deionized water and $1 \mathrm{~g} / \mathrm{L} \mathrm{NaCl}$ solution as feed, respectively. The calculation formulae are shown in equations (1), (2), and (3), respectively [10-12].

$$
\begin{gathered}
\mathrm{A}=\frac{\Delta \mathrm{V}}{\mathrm{A}_{\mathrm{m} \cdot \Delta \mathrm{t} \cdot \mathrm{p}}} \\
\mathrm{B}=\mathrm{A}(\Delta \mathrm{p}-\Delta \pi)\left(\frac{1}{R}-1\right)
\end{gathered}
$$

Where $\Delta \mathrm{V}$ is the increment of the volume in the driving fluid, $\mathrm{L} ; \mathrm{A}_{\mathrm{m}}$ is the effective area of the composite forward osmosis membrane, $\mathrm{m}^{2} ; \Delta \mathrm{t}$ is the time interval; $\mathrm{p}$ is the operating pressure, Bar; $\Delta \mathrm{p}$, $\Delta \pi$ are the pressure difference and osmotic pressure difference on both sides of the composite membrane; $\mathrm{R}$ is the rejection rate of the membrane to the salt solution.

$$
\mathrm{R}=\left(1-\frac{c_{p}}{c_{f}}\right) \times 100 \%
$$

Where $c_{f}$ and $c_{p}$ are the salt concentrations of the feed solution and the exudate, respectively.

The water flux $\mathrm{JV}_{\mathrm{V}}\left[\mathrm{L} /\left(\mathrm{m}^{2} . \mathrm{h}\right)\right]$ and the salt reverse flux $\mathrm{JS}\left[\mathrm{g} /\left(\mathrm{m}^{2} . \mathrm{h}\right)\right]$ of the composite forward osmosis membrane were measured in the AL-FS (separation skin contacting feed liquid) mode. The formulas are shown in equations (4) and (5), respectively [10-12].

$$
\begin{aligned}
\text { Java } & =\frac{\Delta \mathrm{V}}{\mathrm{A}_{\mathrm{m}} \cdot \Delta \mathrm{t}} \\
\text { Jess } & =\frac{\mathrm{c}_{\mathrm{t}} \mathrm{V}_{\mathrm{t}}-\mathrm{c}_{0} \mathrm{~V}_{0}}{\mathrm{~A}_{\mathrm{m}} \cdot \Delta \mathrm{t}}
\end{aligned}
$$

Where $\Delta \mathrm{V}$ is the increment of the volume in the driving fluid, $\mathrm{L} ; \mathrm{A}_{\mathrm{m}}$ is the effective area of the composite forward osmosis membrane, $\mathrm{m}^{2} ; \mathrm{Vt}$ and $\mathrm{c}_{\mathrm{t}}$ are the volume of the raw material liquid and the concentration of solute at the time $\mathrm{t} ; \mathrm{V}_{0}$ and $\mathrm{c}_{0}$ are the volume of raw material liquid and the concentration of solute at the initial time.

In order to characterize the anti-fouling property of forward osmosis membranes before and after grafting modification on positively charged pollutants, the cationic surfactant dodecyl trimethyl ammonium chloride (DTAC) was selected as the positively charged pollutant [8], In the AL-FS mode, the membrane fouling was determined by quantifying the water flux decline in the membrane.

The E. coli was used as model bacteria to evaluate the antibacterial properties of the composite modified membrane. The E. coli broth was diluted to $10^{5} \mathrm{CFU}$ and inoculated onto solid LB medium. The prepared modified composite forward osmosis membrane was then sterilized with the active layer facing down and affixed to the surface of the LB solid medium. The culture medium was placed in a constant temperature incubator at $37^{\circ} \mathrm{C}$ and cultured overnight. The effect was observed after removal on the second day [9]. 


\section{Results and Discussion}

\subsection{Characterization of Membranes.}

As shown in Fig.1, the surface of the aromatic polyamide composite membrane without grafting PEI exhibits a typical "peak-valley" structure, and there is no obvious change in the surface morphology of the two modified membranes after grafting PEI, indicating that the PEI Grafting has no effect on the surface morphology of the membrane. The contact angles of the two modified films using PEI and PEI-AgNPs were $34^{\circ}$ and $37^{\circ}$, respectively, which was lower than the control film of $62^{\circ}$, so hydrophilicity was improved by grafting PEI on the surface.

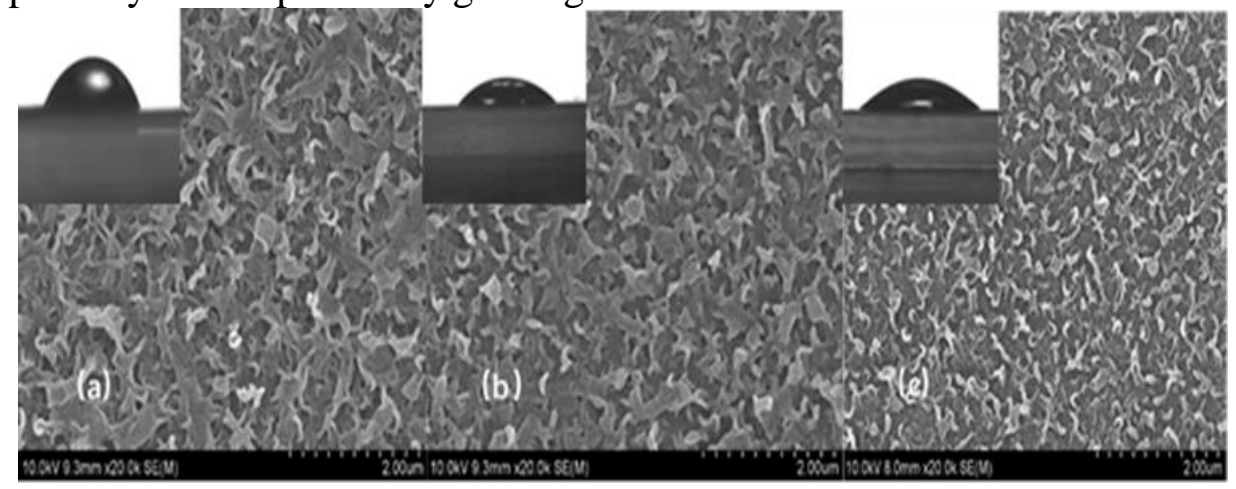

Fig.1 Surface morphology and contact angle of the film (a) Control film (b) PEI modified film (c) PEI-Gaps modified film

As shown in Fig.2, the isoelectric point of the PEI modified membrane and the PEI-AgNPs membrane is at $\mathrm{pH} 6.6$ and $\mathrm{pH} \mathrm{7.4,} \mathrm{respectively,} \mathrm{compared} \mathrm{to} \mathrm{the} \mathrm{isoelectric} \mathrm{point} \mathrm{of} \mathrm{the} \mathrm{Control}$ membrane at $\mathrm{pH}$ 3.3. This is mainly attributed to the successful grafting of PEI, and the introduction of positively charged amine groups which changes the chargeability of the membrane surface. From the viewpoint of film surface charge, PEI molecules successfully grafted onto the surface of the film and caused the film surface charge to change from negative to positive.

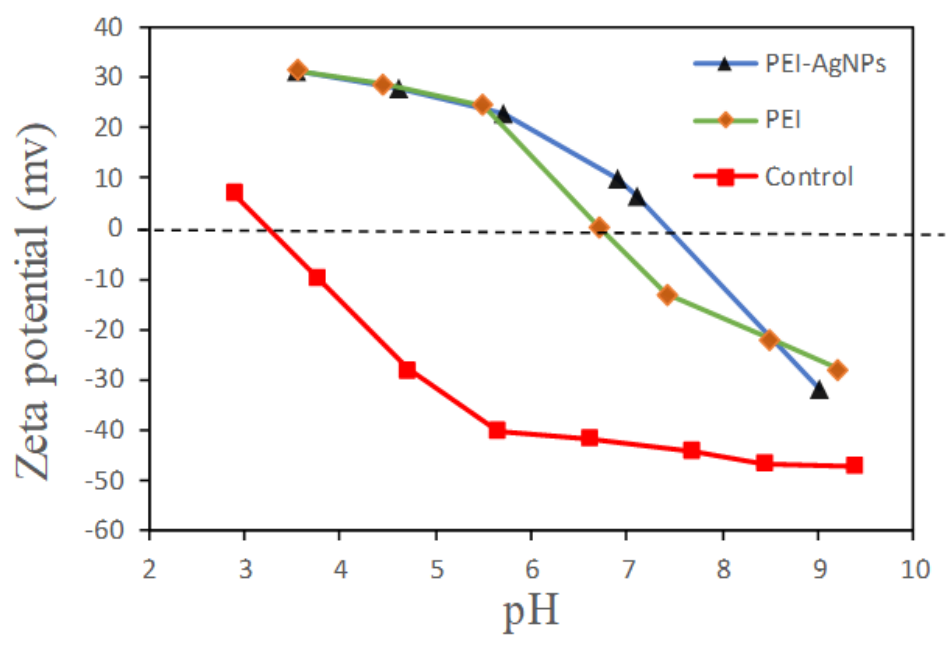

Fig. 2 The zeta potential of the membrane

\subsection{FO Performance.}

As shown in Fig.3, it can be seen from the figure that the water flux of the composite forward osmosis membrane decreases, and the water flux of the Control membrane, PEI modified membrane and PEI-AgNPs membrane were $13.8 \mathrm{~L} /\left(\mathrm{m}^{2} . \mathrm{h}\right), 12.3 \mathrm{~L} /\left(\mathrm{m}^{2} . \mathrm{h}\right)$ and $10.1 \mathrm{~L} /\left(\mathrm{m}^{2} . \mathrm{h}\right)$ in sequence, the salt reverse flux increased were $3.7 \mathrm{~g} /\left(\mathrm{m}^{2} . \mathrm{h}\right), 5.1 \mathrm{~g} /\left(\mathrm{m}^{2} . \mathrm{h}\right)$ and $6.1 \mathrm{~g} /\left(\mathrm{m}^{2} . \mathrm{h}\right)$ respectively. This is because the grafting of PEI leads to an increase in the transmembrane resistance of water, so the water flux of the modified membrane decreased to varying degrees. The change of surface charge properties of the modified membrane may lead to a significant increase in the reverse flux of salt [13]. 


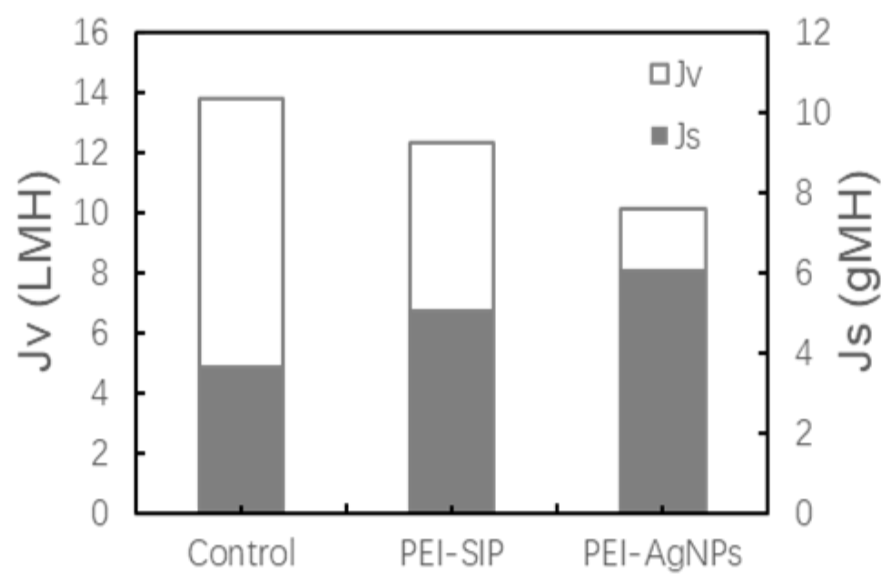

Fig.3 The permeability of different membranes

As shown in Table 1, the composite membranes had higher a values before and after modification, but the modified membrane a values decreased slightly. While value increased. The $\mathrm{NaCl}$ rejection rate of the modified membranes is about $95 \%$, and it shows an upward trend. This is because $\mathrm{Na}^{+}$and $\mathrm{Cl}^{-}$transport are affected by the surface charge of the membrane when it passes through the membrane, and electrostatic repulsion occurs. The effect increases the salt rejection.

Table 1 Intrinsic separation performance of the TFC FO membranes.

\begin{tabular}{cccc}
\hline $\begin{array}{c}\text { FO } \\
\text { membrane }\end{array}$ & $\begin{array}{c}\text { Water permeability,A } \\
(\mathrm{L} / \mathrm{m} 2 . \mathrm{h} . \text { bar })\end{array}$ & $\begin{array}{c}\text { NaCl permeability, B } \\
(\mathrm{L} / \mathrm{m} 2 . \mathrm{h})\end{array}$ & $\begin{array}{c}\text { Salt rejection, } \mathrm{R} \\
(\%)\end{array}$ \\
\hline Control & 2.81 & 0.82 & 94.2 \\
PEI-SIP & 2.54 & 1.36 & 95.1 \\
PEI-AgNPs & 2.32 & 1.27 & 94.8 \\
\hline
\end{tabular}

\subsection{Analysis of Anti-Fouling Performance of PEI Grafting Membrane.}

As shown in Fig.4, the permeation flux of the membrane changes with the contamination time.Dodecyl trimethyl ammonium chloride (DTAC) was used as the simulated pollutant to explore the influence of surface positive charge pollution process on the PEI grafting membrane. As shown in the figure, the water flux of the modified membrane decreased significantly after 300 minutes of operation, while the water flux of the modified membrane was relatively stable. This is because of the introduction of PEI significantly that improves the pollution caused by the adsorption and deposition of positively charged species on the membrane surface.

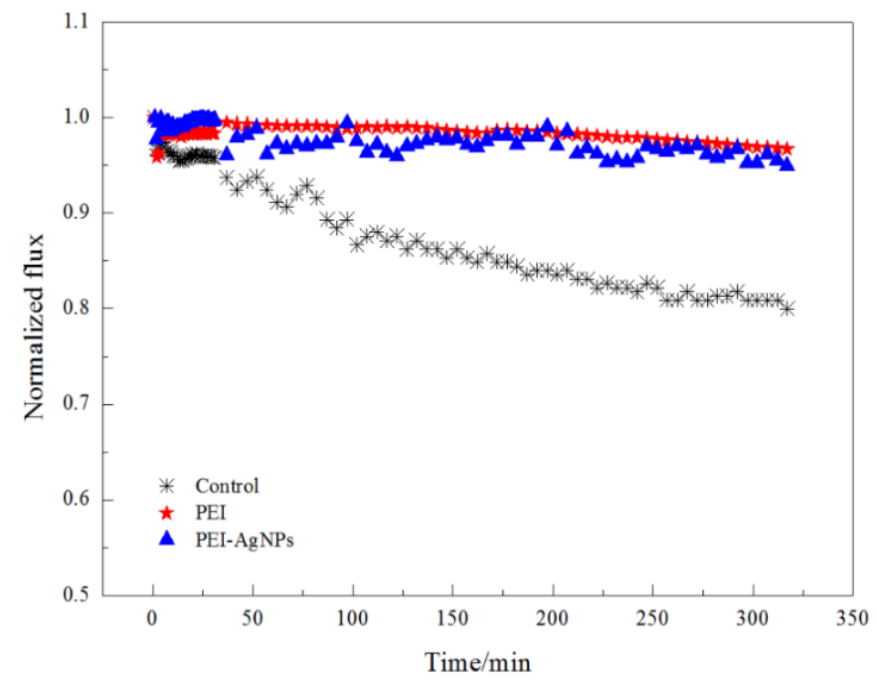

Fig. 4 Effect of cationic surfactant on permeability of control film and PEI grafting Membrane 
Using E. coli as a simulated contaminant, the anti-biofouling performance of the PEI grafting membrane was tested [13-15]. After overnight incubation, removing the membranes on the three different culture media, it shows clearly that there are significant bacterial colony growths under the Control membrane and the PEI membrane. However, PEI-AgNPs membranes shows a smooth media below without signs of bacterial growth. This is mainly due to the excellent bactericidal action of AgNPs, which can inhibit the growth of microorganisms through various pathways.

\section{Conclusion}

In this experiment, Virgin membrane, PEI membrane, PEI-AgNPs membrane were compared for the anti-fouling experiements. The graft modification of PEI significantly improves the hydrophilicity and chargeability of the membrane surface, and the modified membrane has good anti-pollution performance on positively charged cationic surfactants. And after the introduction of $\mathrm{Ag}$ nanoparticles, the modified membrane showed excellent antibacterial properties.

\section{Acknowledgments}

The authors would like to thank the partial financial support from the Fundamental Research Funds for the Central Universities (2232018D3-09) and National Natural Science Foundation of China $(21507142,21477018)$.

\section{References}

[1]. Valla dares, L.R., et al., Life cycle cost of a hybrid forward osmosis - low pressure reverse osmosis system for seawater desalination and wastewater recovery. Water Research, 2016. 88: p. 225.

[2]. Li, Z., et al., Calcium carbonate scaling in seawater desalination by ammonia-carbon dioxide forward osmosis: Mechanism and implications. Journal of Membrane Science, 2015. 481: p. 36-43.

[3]. Valla dares, L.R., et al., Forward osmosis niches in seawater desalination and wastewater reuse. Water Research, 2014. 66: p. 122-139.

[4]. Zhao, P., et al., The performance of forward osmosis process in treating the surfactant wastewater: The rejection of surfactant, water flux and physical cleaning effectiveness. Chemical Engineering Journal, 2015. 281: p. 688-695.

[5]. Yu, et al., Surface modification of thin-film composite polyamide reverse osmosis membranes with thermo-responsive polymer (TRP) for improved fouling resistance and cleaning efficiency. Separation \& Purification Technology, 2011. 76(3): p. 283-291.

[6]. She, Q., et al., Membrane fouling in osmotically driven membrane processes: A review. Journal of Membrane Science, 2016. 499: p. 201-233.

[7]. Lee, S., et al., Comparison of fouling behavior in forward osmosis (FO) and reverse osmosis (RO). Journal of Membrane Science, 2010. 365(1): p. 34-39.

[8]. $\mathrm{Cu}$, J., et al., Positively charged aromatic polyamide reverse osmosis membrane with high anti-fouling property prepared by polyethylenimine grafting. Desalination, 2015. 365: p. 398-406.

[9]. Yin, J., et al., Attachment of silver nanoparticles (AgNPs) onto thin-film composite (TFC) membranes through covalent bonding to reduce membrane biofouling. Journal of Membrane Science, 2013. 441(16): p. 73-82. 
[10]. Chen, G., et al., Concentrating underground brine by FO process: Influence of membrane types and spacer on membrane scaling. Chemical Engineering Journal, 2016. 285: p. 96-100.

[11]. Chen, G., et al., Treatment of shale gas drilling flowback fluids (SGDFs) by forward osmosis: Membrane fouling and mitigation. Desalination, 2015. 366: p. 113-120.

[12]. Xiao, P., et al., A sacrificial-layer approach to fabricate polysulfone support for forward osmosis thin-film composite membranes with reduced internal concentration polarisation. Journal of Membrane Science, 2015. 481: p. 106-114.

[13]. Dong, C., et al., A green strategy to immobilize silver nanoparticles onto reverse osmosis membrane for enhanced anti-biofouling property. Desalination, 2016. 401: p. 32-41.

[14]. Qi, W.Z., et al., Antimicrobial Membrane Surfaces via Efficient Polyethyleneimine Immobilization and Cationization. Applied Surface Science, 2017. 426.

[15]. Ma, W., et al., Spray- and spin-assisted layer-by-layer assembly of copper nanoparticles on thin-film composite reverse osmosis membrane forÂ biofouling mitigation. Water Research, 2016. 99: p. 188-199. 